\title{
COVID-19 PANDEMIC AND VACCINE
}

\author{
Dr. Santosh Shah \\ Department of General Surgery (CTVS) \\ Universal College of Medical Sciences, Bhairahawa, Nepal
}

COVID-19, caused by SARS-CoV-2, was first reported in Wuhan, in December 2019 and later spread globally. The World Health Organization (WHO) declared the outbreak a public health emergency of international concern (PHEIC) on 30 January and a pandemic on 11 March 2020. ${ }^{1}$ In Nepal, the first case was registered on $3^{\text {rd }}$ January 2020 . Since then, there have been 591,494 confirmed cases of COVID-19 with 7,990 deaths. $^{2}$

In the second wave of Covid-19, from March 2021, South Asia seems to have turned into the epicentre as most of the countries in the region, including India, Nepal, Bangladesh, and Pakistan have been badly infected by the coronavirus. Instead of being controlled, the situation is getting more flared up as each day passes. Of late, the situation in Nepal is gradually becoming alarming as two out of five people tested return positive. Subsequently, Nepal started to face shortage of oxygen, ventilators, and hospital beds required for the treatment of severe cases. Medicines like Remdesivir and other medical equipment are sold at exorbitant prices.

As COVID-19 infections surge across the country, the most promising solution to contain the raging pandemic is to accelerate vaccination. Approved COVID-19 vaccines provide a high degree of protection against getting seriously ill and dying from the disease, although no vaccine is $100 \%$ protective. $^{3}$ Thus, in addition to vaccination one must follow the other preventive measures of staying at least 1 metre away from others, covering a cough or sneeze in your elbow, frequently cleaning your hands, wearing a mask and avoiding poorly ventilated rooms or opening a window. ${ }^{3}$

Currently, there are three main types of COVID-19 vaccines that are authorized and recommended or undergoing largescale (Phase 3 ) clinical trials. ${ }^{4}$ First, mRNA vaccines which contain material from the virus that causes COVID-19 that gives our cells instructions for how to make a harmless protein that is unique to the virus. After our cells make copies of the protein, they destroy the genetic material from the vaccine. Our bodies recognize that the protein should not be there and build T-lymphocytes and B-lymphocytes that will remember how to fight the virus that causes COVID-19 if we are infected in the future. Second, Protein subunit vaccines include harmless protein portion of the virus that causes COVID-19 instead of the entire germ. Once vaccinated, our bodies recognize that the protein should not be there and build T- lymphocytes and antibodies to fight COVID-19 in the future. Third is Vector vaccines contain a modified version of a different virus than the one that causes COVID-19. Inside the shell of the modified virus, there is material from the virus that causes COVID-19. This is called a "viral vector." Once the viral vector is inside our cells, the genetic material gives cells instructions to make a protein that is unique to the virus that causes COVID-19. Using these instructions, our cells make copies of the protein. This prompts our bodies to build Tlymphocytes and B-lymphocytes that will remember how to fight that virus if we are infected in the future. ${ }^{4}$

As of 3 June 2021, WHO has evaluated several vaccines against COVID-19, have met the necessary criteria for safety and efficacy. First is AZD1222 (Covishield and Vaxzevria), viral vector vaccine developed by AstraZeneca and Oxford university which has an efficacy of $63.09 \%$ against symptomatic SARS-CoV-2 infection. The recommended dosage is two doses given intramuscularly $(0.5 \mathrm{ml}$ each $)$ with an interval of 8 to 12 weeks. Longer dose intervals within the 8 to 12 weeks range are associated with greater vaccine efficacy. ${ }^{5}$ It is an Adenovirus vaccine. Second is mRNA-1273 vaccine developed by Moderna in United States, has an efficacy of approximately $92 \%$ in protecting against COVID19, starting 14 days after the first dose. WHO Strategic Advisory Group of Experts (SAGE) on Immunisation recommends the use of the Moderna mRNA-1273 vaccine at a schedule of two doses $(100 \mu \mathrm{g}, 0.5 \mathrm{ml}$ each) 28 days apart. If necessary, the interval between the doses may be extended to 42 days. It is mRNA-based vaccine. ${ }^{6}$

Third is BNT162b2, mRNA vaccine developed by Pfizer and BioNTech in United States, has an efficacy of $95 \%$ against symptomatic SARS-CoV-2 infection. WHO has thoroughly assessed the quality, safety, and efficacy of the vaccine and has recommended its use for persons above the age of 16 . A protective effect starts to develop 12 days after the first dose, but full protection requires two doses which WHO recommends be administered with a 21 to 28-day interval. It is mRNA-based vaccine. ${ }^{7}$ Fourth is Janssen Vaccines (Johnson \& Johnson), viral vector vaccine which has an efficacy of $85.4 \%$ against severe disease and hospitalization. SAGE recommends the use of Janssen Ad26.CoV2.S as one dose ( 0.5 $\mathrm{ml}$ ) given intramuscularly and vaccine is not recommended for persons younger than 18 years of age. It is non-replicating 
viral vector type vaccine. ${ }^{8}$ Fifth is Sinopharm COVID-19 vaccine (inactivated virus), developed by China, have an efficacy of $79 \%$ against symptomatic SARS-CoV-2 infection 14 or more days after the second dose. SAGE recommends the use of Beijing Institute of Biological Products (BIBP) vaccine as 2 doses $(0.5 \mathrm{ml})$ given intramuscularly for people aged 18 and above. WHO recommends an interval of 3-4 weeks between the first and second dose. It is an inactivated vaccine. ${ }^{9}$ Sixth is Sinovac COVID-19 vaccine (inactivated virus), also developed by China, has an efficacy of $51 \%$ against symptomatic SARS-CoV-2 infection, $100 \%$ against severe COVID-19, and $100 \%$ against hospitalization starting 14 days after receiving the second dose. SAGE recommends the use of Sinovac-CoronaVac vaccine as 2 doses $(0.5 \mathrm{ml})$ given intramuscularly for people aged 18 and above. WHO recommends an interval of 2-4 weeks between the first and second dose. It is recommended that all vaccinated individuals receive two doses. ${ }^{10}$ It is also an inactivated vaccine (formalin with alum adjuvant).

In addition to these vaccines, there are several other authorized/approved vaccines such as Sputnik V (a recombinant adenovirus vaccine developed by Gamaleya Research Institute Russia), Covaxin (an inactivated vaccine, developed by BBIL India), QazVac (an inactivated vaccine developed by Kazakhstan Research Institute of Biological Safety Problems, Kazakhstan). ${ }^{11}$

The COVID-19 vaccines are safe for most people 18 years and older, including those with pre-existing conditions of any kind, including auto-immune disorders. These conditions include hypertension, diabetes, asthma, pulmonary, liver and kidney disease, as well as chronic infections that are stable and controlled. $^{3}$ Pregnant women at high risk of exposure to SARS-CoV-2 (e.g., health workers) or who have comorbidities which add to their risk of severe disease, may be vaccinated in consultation with their health care provider. ${ }^{7}$ There is not yet enough evidence on the use of vaccines against COVID-19 in children to make recommendations for children to be vaccinated against COVID-19.

Different types of vaccines work in different ways to offer protection. But with all types of vaccines, the body is left with a supply of "memory" T-lymphocytes as well as Blymphocytes that will remember how to fight that virus in the future. It typically takes a few weeks after vaccination for the body to produce T-lymphocytes and B-lymphocytes. Therefore, it is possible that a person could be infected with the virus that causes COVID-19 just before or just after vaccination and then get sick because the vaccine did not have enough time to provide protection. ${ }^{4}$ Common side effects after vaccination, which indicate that a person's body is building protection to COVID-19 infection include arm soreness, mild fever, tiredness, headaches, muscle, or joint aches. If you experience an immediate severe allergic reaction to a first dose of the COVID-19 vaccine, you should not receive additional doses of the vaccine. It's extremely rare for severe health reactions to be directly caused by vaccines. Taking painkillers such as paracetamol before receiving the COVID-19 vaccine to prevent side effects is not recommended. This is because it is not known how painkillers may affect how well the vaccine works. However, you may take paracetamol or other painkillers if you do develop side effects such as pain, fever, headache, or muscle aches after vaccination.

Nepal was one of the first few countries in the world that initiated the inoculation drive as early as January 27 with Covishield vaccine donated by Government of India this year but there is great shortage of vaccine. The country also received 348,000 doses of Covishield under the World Health Organisation backed COVAX facility on March 7. ${ }^{12}$ Chinese government has given Nepal 1.8 million doses of the Sinopharm vaccine in grants 800,000 doses in March and an additional one million doses in early June. ${ }^{13}$

As of 29 May 2021, a total of 2,802,596 vaccine doses have been administered in Nepal. ${ }^{2}$ Although, Nepal required a total supply of 44 million vaccines to inoculate 22 million of its eligible population. It needs to procure the vaccines from the international market for which it might have to pay more. But people's lives are more important than the cost of the vaccines.

\section{REFERENCES}

1. Rayamajhee B, Pokhrel A, Syangtan G, Khadka S, Lama B, Rawal LB, Mehata S, Mishra SK, Pokhrel R, Yadav UN. How Well the Government of Nepal Is Responding to COVID-19? An Experience From a Resource-Limited Country to Confront Unprecedented Pandemic. Frontiers in Public Health. 2021 Feb $17 ; 9: 85$

2. World health organization. Nepal Situation. [Online]. Available from: https://covid19.who.int/region/searo/country/np

3. World health organization. COVID-19 advice for the public: Getting vaccinated. Available from: https://www.who.int/ emergencies/diseases/novel-coronavirus-2019/covid-19vaccines/advice

4. Center for disease control and prevention. Different COVID-19 Vaccines. Available from: https://www.cdc.gov/coronavirus /2019-ncov/vaccines/different-vaccines

5. World health organization. The Oxford/AstraZeneca COVID19 vaccine: what you need to know. Available from: https://www.who.int/news-room/feature-stories/detail/theoxford-astrazeneca-covid-19-vaccine-what-you-need-to-know 
6. World health organization. The Moderna COVID-19 (mRNA1273) vaccine: what you need to know. Available from: https://www.who.int/news-room/feature-stories/detail/themoderna-covid-19-mrna-1273-vaccine-what-you-need-toknow

7. World health organization. The Pfizer BioNTech COVID-19 vaccine: What you need to know. Available from: https://www. who.int/news-room/feature-stories/detail/who-can-take-thepfizer-biontech-covid-19-vaccine

8. World health organization. The J\&J COVID-19 vaccine: What you need to know.Available from: https://www.who.int/newsroom/feature-stories/detail/the-j-j-covid-19-vaccine-whatyou-need-to-know

9. World health organization. The Sinopharm COVID-19 vaccine: What you need to know. Available from: https://www.who.int /news-room/feature-stories/detail/the-sinopharm-covid-19vaccine-what-you-need-to-know

10. World health organization. The Sinovac COVID-19 vaccine: What you need to know. Available from: https://www.who.int/ news-room/feature-stories/detail/the-sinopharm-covid-19vaccine-what-you-need-to-know

11. Crave J. COVID-19 vaccine tracker. Available from: https:// www. raps.org/news-and-articles/news-articles/2020/3/ covid19-vaccine-tracker

12. Jha HB. Second Wave of COVID-19 getting out of control in Nepal. Available from: https://www.orfonline.org/expertspeak/second-wave-of-covid-19-getting-out-of-control-innepal

13. Sharma G. China to gift 1 million COVID-19 vaccine doses to Nepal. Available from: https://www.reuters.com/business/ healthcare-pharmaceuticals/china-gift-1-million-covid-19vaccine-doses-nepal-2021-05-26 